\title{
Educational nurse-led telephone intervention shortly before colonoscopy as a salvage strategy after previous bowel preparation failure: a multicenter randomized trial
}

\section{(ㄷ)(우)}

\section{Authors}

Marco Antonio Alvarez-Gonzalez 1,2,3, Miguel Ángel Pantaleón Sánchez¹,2, Belén Bernad Cabredo4, Ana GarcíaRodríguez $^{5}$, Santiago Frago Larramona ${ }^{6}$, Oscar Nogales ${ }^{7}$, Pilar Díez Redondo ${ }^{8}$, Ignasi Puig del Castillo ${ }^{9}$, Cristina Romero Mascarell ${ }^{10}$, Noemí Caballero ${ }^{11}$, Iván Romero Sánchez-Miguel ${ }^{12}$, Rocío Pérez Berbegal1 , Domingo Hernández Negrín ${ }^{13}$, Gema Bujedo Sadornill ${ }^{4}$, Alicia Pérez Oltra ${ }^{5}$, Gemma Casals Urquiza ${ }^{1}$, Jaume Amorós Martínez ${ }^{14}$, Agustín Seoane Urgorri ${ }^{1,3}$, Inés Ana Ibáñez Zafón¹, Antonio Z. Gimeno-García ${ }^{13}$

Institutions

1 Department of Digestive Diseases, Hospital del Mar, Barcelona, Spain

2 Department of Medicine, Universitat Autònoma de Barcelona, Barcelona, Spain

3 IMIM, Hospital del Mar Medical Research Institute, Barcelona, Spain

4 Department of Digestive Diseases, Hospital Universitario de Burgos, Burgos, Spain

5 Department of Digestive Diseases, Hospital de Viladecans, Barcelona, Spain

6 Department of Digestive Diseases, Hospital Santa Bárbara, Soria, Spain

7 Department of Digestive Diseases, Hospital Gregorio Marañón, Madrid, Spain

8 Department of Digestive Diseases, Hospital del Rio Hortega, Valladolid, Spain

9 Department of Digestive Diseases, Altahia Xarxa Asistencial Universitaria de Manresa, Barcelona, Spain

10 Department of Digestive Diseases, Consorci Sanitari de Terrassa, Barcelona, Spain

11 Department of Digestive Diseases, Hospital Germans Trias i Pujol, Badalona, Spain

12 Department of Digestive Diseases, Hospital 12 de Octubre, Madrid, Spain

13 Department of Digestive Diseases, Hospital Universitario de Canarias, La Laguna, Spain

14 Open University of Catalonia, Barcelona, Spain

submitted 24.12.2019

accepted after revision 22.4.2020

published online 17.6 .2020

Bibliography

Endoscopy 2020; 52: 1026-1035

DOI 10.1055/a-1178-9844

ISSN 0013-726X (c) 2020. The Author(s).

This is an open access article published by Thieme under the terms of the Creative Commons Attribution-NonDerivative-NonCommercial License, permitting copying and reproduction so long as the original work is given appropriate credit. Contents may not be used for commecial purposes, or adapted, remixed, transformed or built upon. (https://creativecommons.org/licenses/by-nc-nd/4.0/)

\section{GRAPHICAL ABSTRACT}

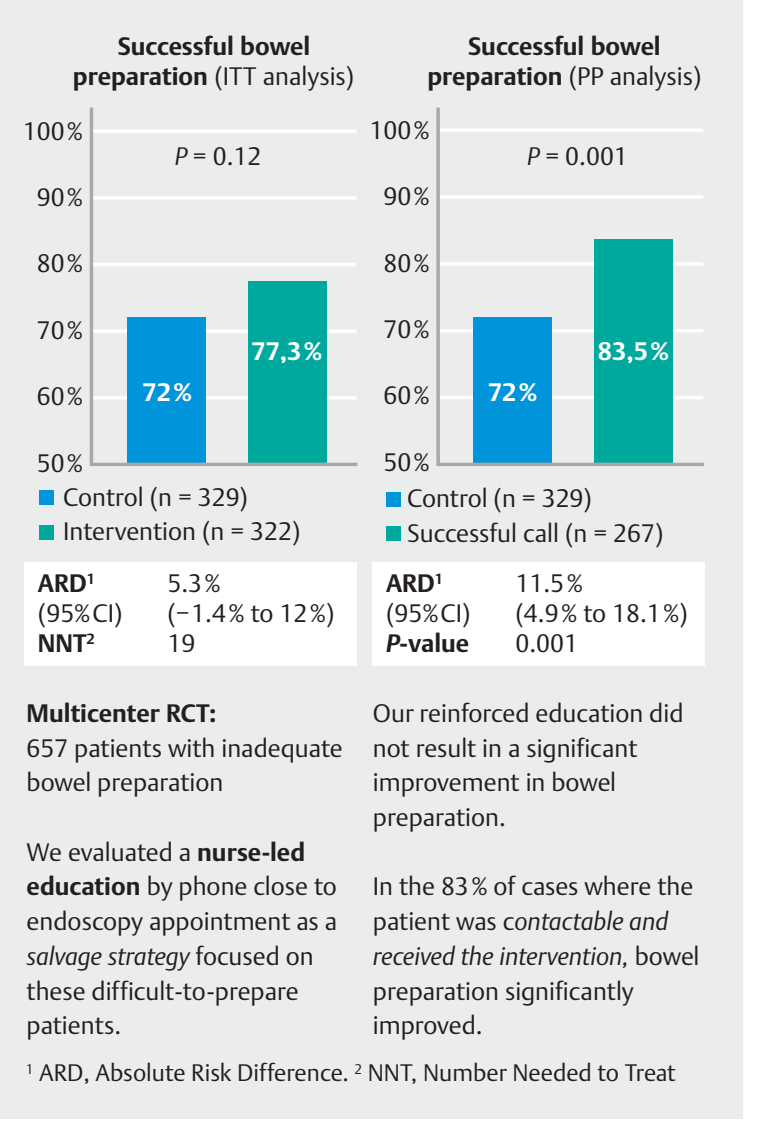


Supplementary material

Online content viewable at:

https://doi.org/10.1055/a-1178-9844

Scan this QR-Code for the author commentary.

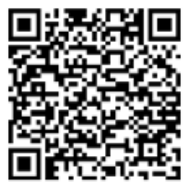

Corresponding author

Marco A. Álvarez-González, MD, PhD, Department of Digestive Diseases, Hospital del Mar de Barcelona, Passeig Marítim de la Barceloneta, 25-29, 08003 Barcelona, Spain Fax: +34-93-2483254

marcoalgo@gmail.com

\section{ABSTRACT}

Background The most important predictor of unsuccessful bowel preparation is previous failure. For those patients with previous failure, we hypothesized that a nurse-led educational intervention by telephone shortly before the colonoscopy appointment could improve cleansing efficacy.
Methods We performed a multicenter, endoscopist-blinded, randomized controlled trial. Consecutive outpatients with previous inadequate bowel preparation were enrolled. Both groups received the same standard bowel preparation protocol. The intervention group also received reinforced education by telephone within 48 hours before the colonoscopy. The primary outcome was effective bowel preparation according to the Boston Bowel Preparation Scale. Intention-to-treat (ITT) analysis included all randomized patients. Per-protocol analysis included patients who could be contacted by telephone and the control cases.

Results 657 participants were recruited by 11 Spanish hospitals. In the ITT analysis, there was no significant difference between the intervention and control groups in the rate of successful bowel preparation $(77.3 \%$ vs. $72 \% ; P=0.12)$. In the intervention group, 267 patients $(82.9 \%)$ were contacted by telephone. Per-protocol analysis revealed significantly improved bowel preparation in the intervention group (83.5\% vs. $72.0 \% ; P=0.001)$.

Conclusion Among all patients with previous inadequate bowel preparation, nurse-led telephone education did not result in a significant improvement in bowel cleansing. However, in the $83 \%$ of patients who could be contacted, bowel preparation was substantially improved. Phone education may therefore be a useful tool for improving the quality of bowel preparation in those cases.

\section{Introduction}

The quality of bowel preparation represents a key factor affecting the efficacy of colonoscopy. The European Society of Gastrointestinal Endoscopy recently raised the quality threshold for adequate bowel preparation to $\geq 90 \%$ [1]. However, in clinical practice, between $20 \%$ and $30 \%$ of patients do not achieve successful bowel preparation [2]. Inadequate bowel cleansing results in harmful consequences, such as aborted procedures, missed lesions, diagnostic delays, and increased risks and healthcare costs [3,4]. A history of unsuccessful bowel preparation represents the most relevant predictor for inadequate bowel preparation [5]. A retrospective study showed that more than $23 \%$ of patients with previous inadequate bowel preparation also failed to achieve successful bowel cleansing in repeat procedures and could be considered a difficult-to-treat patient group $[2,6,7]$. Unfortunately, the best approach for patients with failed bowel preparation has not yet been determined [8].

Modifiable and nonmodifiable factors affect the quality of bowel preparation. Among the modifiable risk factors, patient adherence to instructions plays a leading role in achieving adequate bowel cleansing [9]. Few medical explorations require so rigorous and complex a preparation as bowel cleansing, so it may be considered a major barrier for some patients undergoing colonoscopy [10].

In recent years, multiple strategies to reinforce patient education have demonstrated improved bowel cleansing quality, such as visual aids, face-to-face sessions, telephone and short message service (SMS) communications, and smartphone and social media applications [11 - 13]. International guidelines [8, 14] recommend the use of enhanced instructions for bowel preparation, although there is no consensus on which educational tool is the best or how to implement it. Furthermore, there are no studies that specifically address the usefulness of repeated instructions in patients after previous bowel preparation failure; reinforced education for bowel preparation may represent an effective tool to improve compliance with instructions in these patients.

The aim of this study was to evaluate the effectiveness of a nurse-led educational intervention by telephone shortly before the colonoscopy appointment as a salvage strategy to improve bowel cleansing after previous bowel preparation failure.

\section{Methods}

\section{Study design}

A prospective, multicenter, endoscopist-blinded, randomized controlled trial (RCT) was conducted to determine whether an educational intervention may increase bowel preparation success after previous bowel preparation failure. The study was performed between January 2017 and June 2018, and was conducted in 11 tertiary hospitals in Spain, with the Hospital del Mar of Barcelona being the coordinating center. The study protocol was approved by the ethics committee of the coordinating center $(6605 / \mathrm{l})$ and the recruiting centers. We followed the Declaration of Helsinki ethical guidelines and registered 
the study protocol at ClinicalTrials.gov (NCT03055689). Written informed consent was obtained from all enrolled patients.

\section{Study population, treatment allocation, and masking}

Consecutive outpatients aged $18-85$ years with previous inadequate bowel cleansing according to the Boston Bowel Preparation Scale (BBPS) [15] were invited to participate. We included patients with any indication for colonoscopy, such as surveillance, diagnosis, and screening. Exclusion criteria included the inability to follow instructions or to use a telephone, including hearing problems, unwillingness to participate, severe renal impairment, active inflammatory bowel disease, pregnancy or breastfeeding.

Consenting patients were randomized into blocks of six individuals in each center using a computer-generated block randomization table with a 1:1 allocation rate to the control group and the intervention group. A colonoscopy appointment was scheduled for within 3 months. A research nurse provided bowel preparation instructions and self-administered questionnaires. The patients were asked to provide at least two telephone numbers for themselves or their family members so that the nurses could contact them. In all case, the nurse-led education was always addressed to the patient directly.

Skilled endoscopists (>1000 colonoscopies each) who were blinded to the randomization rated the bowel cleansing using the BBPS. Before starting the study, all endoscopists underwent a calibration exercise to improve consensus and minimize interobserver variability. All colonoscopies were performed under deep sedation. Following guideline recommendations, sedation was administered by a gastroenterologist, or by an anesthesiologist in patients with risk factors [16].

\section{Standard bowel preparation protocol}

Both groups received the same bowel preparation protocol based on international guideline recommendations $[14,17]$. First, all participants received standard education, which consisted of a face-to-face visit by an endoscopy nurse who provided verbal and written instructions detailed in a booklet. The booklet was the same for all centers and was written in plain language and with some pictures to facilitate the understanding of the instructions. Second, a low-fiber diet was implemented 7 days before the colonoscopy, as this diet has been shown to be better tolerated and may be more effective than a clear liquid diet [18]. We also recommended a discontinuation of oral iron treatment 7 days before the colonoscopy. Finally, a split-dose laxative regimen was instructed, with the second dose starting 5 hours before the colonoscopy. A total of $4 \mathrm{~L}$ of polyethylene glycol (PEG) was chosen as the laxative, according to a previous publication [19].

\section{Reinforced educational intervention}

In addition to the standard bowel preparation protocol, the intervention group received reinforced education via a nurse-led telephone call within 24 - 48 hours prior to the colonoscopy appointment. To ensure consistency of the intervention, all calls were centralized at the coordinating center (Hospital del Mar de Barcelona) and were conducted by two trained endoscopy nurses.

The main purpose of the telephone intervention was to reinforce the instructions of the bowel preparation based on three aims: 1) to ensure compliance with the low-fiber diet and the laxative intake protocol in terms of both timing and dose; 2) to emphasize the importance of properly performing the bowel preparation protocol to guarantee adequate visualization and detection of concerning lesions; and 3) to clarify any patient doubts about the bowel preparation protocol. The content of the call was the same as the standard education given in the booklet to both groups (see Appendix 1s in the online-only supplementary material).

\section{Outcome measures}

The primary outcome was the rate of successful bowel preparation, as measured by the BBPS [20]. Success was defined as all colon segments scoring $\geq 2$ points. Missing efficacy data due to nonattendance at the colonoscopy appointment were imputed as bowel preparation failures. In patients with partial colectomy or an incomplete colonoscopy due to a stricture, bowel preparation success was defined when all evaluable segments had $\geq 2$ points. Similarly, bowel preparation failure was recorded when one or more segments had a rating $<2$ points.

Secondary end points included the BBPS scale for patients in whom the colonoscopy was performed, complete colonoscopy rate, overall and proximal (to the splenic flexure) colon lesions detected, such as adenomas and serrated lesions. Additional secondary outcomes, which were planned a priori but not listed in the registered protocol at ClinicalTrials.gov, were attendance at the colonoscopy appointment, adherence to the diet and laxative intake, cancer, mean adenoma per patient, and adenomas or serrated lesions in the distal colon.

\section{Data collection}

We recorded variables known to potentially impact bowel cleansing [2, 7, 21, 22]. Before the colonoscopy, all participants completed self-administered questionnaires relating to the diet and laxative intake protocol.

At the colonoscopy appointment, an investigator collected the questionnaires. After the procedure, endoscopists, who were blinded to the intervention allocation, registered the bowel cleansing quality and any relevant information regarding detected lesions. Research electronic data capture (REDcap), a secure web application, was used to collect and manage all data collected from the 11 hospitals.

\section{Statistical analysis}

The sample size was estimated to demonstrate the superiority of the educational intervention. Using an estimated bowel preparation success rate of $70 \%$ for the control group, we calculated a $10 \%$ improvement with the intervention, with an $\alpha$ risk of $0.05,80 \%$ power, and a dropout rate of $10 \%$, resulting in 326 patients per arm.

The intention-to-treat (ITT) analysis included all randomized patients. The per-protocol analysis compared outcomes in pa- 


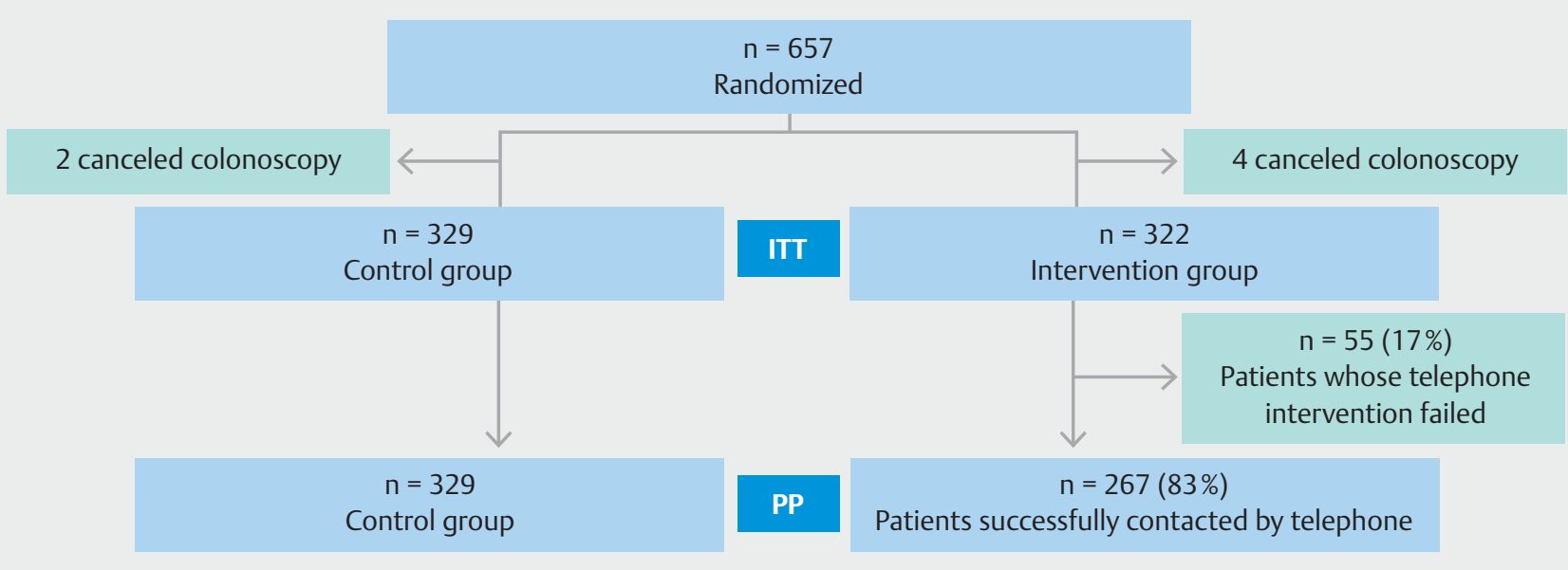

- Fig. 1 Flow-chart of the study. ITT, intention to treat; PP, per protocol.

tients who were successfully contacted by telephone and the control group.

Three post-hoc analyses were conducted. First, we analyzed bowel preparation success according to several risk factors; Bonferroni correction was performed for significant $P$ values. Second, we analyzed telephone contact availability in the intervention group. Finally, an economic analysis was developed to quantify the direct costs of the telephone intervention compared with the cost of an unsuccessful bowel preparation. The average cost of the telephone intervention was based on the 2019 Hospital del Mar collective agreement. The hourly cost of a trained nurse was $€ 33$. We assumed that an unsuccessful bowel preparation in a public health system would result in the loss of opportunity for a valid colonoscopy. The cost of a colonoscopy was obtained from the Public Health System of Catalonia [23] and was estimated to be in $€ 320$.

The qualitative variables were compared between groups by the Pearson's chi-squared test or Fisher's exact test if applicable. Continuous variables are expressed as the means with $95 \%$ confidence intervals ( $\mathrm{Cls}$ ) and were compared using Student's $t$ test. Two-tailed $P$ values of $<0.05$ were considered statistically significant. Stata software version 15.1 (StataCorp LLC, College Station, Texas, USA) was used by our research statistician to perform the analysis.

\section{Results}

\section{Patient characteristics}

A total of 657 outpatients with previous inadequate bowel preparation were recruited. After randomization, six patients canceled their colonoscopy appointment. Finally, 329 individuals in the control group and 322 in the intervention group were included in the ITT analysis. The research nurses successfully contacted 267 (82.9\%) patients in the telephone group and these patients were included in the per-protocol analysis ( $>$ Fig. 1).

Comparison of the baseline characteristics between the control and intervention groups showed an imbalance in the Amer- ican Society of Anesthesiologists score, with more class I patients in the control group. There were no significant differences between the two groups in terms of the initial failed colonoscopy ( $\triangleright$ Table 1$)$. The median waiting time from the scheduling visit to the colonoscopy was 30 days in both groups.

\section{Primary outcome}

In the ITT analysis, the rate of successful bowel preparation was not significantly higher in the telephone group than in the control group (77.3\% vs. $72.0 \%$; $P=0.12$ ); the absolute risk difference (ARD) was $5.3 \%(95 \% \mathrm{Cl}-1.4 \%$ to $12.0 \%)$ and the number needed to treat (NNT) was 18.9 cases ( $>$ Table 2 ).

The per-protocol analysis revealed a significantly higher bowel preparation success rate in the intervention group ( $83.5 \%$ vs. $72.0 \%$; $P=0.001)$, with an ARD of $11.5 \%(95 \% \mathrm{Cl} 4.9 \%$ to $18.1 \%)$ ( Table 2).

\section{Secondary outcomes}

In the ITT analysis, there were no significant differences between the groups in the colonoscopy attendance rate. Bowel cleansing adequacy in patients who underwent colonoscopy was not significantly different between the groups for all three colon segments or in the right colon, but there was a trend toward better cleansing adequacy in the transverse and left colon in the telephone group. There was also a trend toward a better complete colonoscopy rate in the telephone group. There were no differences in adherence to the diet, laxative intake or the interval between the last dose and the colonoscopy between the groups ( $\triangleright$ Table 2 ).

In the per-protocol analysis, there was significantly better colonoscopy attendance in the telephone group.Segmental bowel cleansing was significantly better in the left colon, and there was a trend toward more adequate cleansing globally and in the right and transverse colon. There was also a significantly higher rate of a complete colonoscopy.

There were no differences in the adenoma detection rate, but there were more patients with multiple adenomas and ser- 
- Table 1 Baseline characteristics of the patients regarding the initial failed colonoscopy.

\begin{tabular}{|c|c|c|c|}
\hline Variable & $\begin{array}{l}\text { Control } \\
(n=329)\end{array}$ & $\begin{array}{l}\text { Telephone } \\
(\mathrm{n}=322)\end{array}$ & $\begin{array}{l}P \\
\text { value }\end{array}$ \\
\hline Interval, median (IQR), days & $30(44.5)$ & $30(39)$ & 0.92 \\
\hline Age, median (IQR), years & $63.7(17.6)$ & $64.4(15.7)$ & 0.38 \\
\hline Male sex, n (\%) & $185(56.2)$ & $179(55.6)$ & 0.87 \\
\hline BMI, median (IQR) & $27.1(5.3)$ & $26.7(7.1)$ & 0.93 \\
\hline Diabetes mellitus, n (\%) & $79(24.0)$ & $61(18.9)$ & 0.12 \\
\hline $\begin{array}{l}\text { Abdominal/pelvic surgery, } \\
\mathrm{n}(\%)\end{array}$ & $138(41.9)$ & $135(41.9)$ & 0.99 \\
\hline Partial colectomy, n (\%) & $35(10.6)$ & $30(9.3)$ & 0.52 \\
\hline Constipation, n (\%) & $123(37.4)$ & $125(38.8)$ & 0.78 \\
\hline $\begin{array}{l}\text { Tricyclic antidepressants, } \\
\mathrm{n}(\%)\end{array}$ & $28(8.5)$ & $32(9.9)$ & 0.53 \\
\hline Calcium blockers, n (\%) & $27(8.2)$ & $20(6.2)$ & 0.33 \\
\hline Opiates, n (\%) & $14(4.3)$ & $17(5.3)$ & 0.54 \\
\hline Illiteracy, n (\%) & $7(2.1)$ & $6(1.9)$ & 0.81 \\
\hline \multicolumn{3}{|l|}{ ASA class, n (\%) } & 0.04 \\
\hline .1 & $133(40.4)$ & $100(31.1)$ & \\
\hline . II & $171(52.0)$ & $193(59.9)$ & \\
\hline . III & $25(7.6)$ & $29(9.0)$ & \\
\hline \multicolumn{3}{|l|}{ Indication, n (\%) } & 0.81 \\
\hline - Screening & $87(26.4)$ & $85(26.4)$ & \\
\hline - Surveillance & $96(29.2)$ & $95(29.5)$ & \\
\hline - Diagnostic & $145(44.1)$ & $142(44.1)$ & \\
\hline \multicolumn{3}{|l|}{ Referring physician, n (\%) } & 0.31 \\
\hline - Gastroenterologist & $76(23.1)$ & $74(23.0)$ & \\
\hline - Primary care & $187(56.8)$ & $199(61.8)$ & \\
\hline - Other specialties & $65(19.8)$ & $49(15.2)$ & \\
\hline First colonoscopy, n (\%) & $143(43.5)$ & $155(48.1)$ & 0.13 \\
\hline \multicolumn{3}{|l|}{ Laxative, n (\%) } & 0.51 \\
\hline - MCSP & $94(28.6)$ & $87(27.0)$ & \\
\hline - PEG + ascorbate, 2 L & $99(30.1)$ & $82(25.5)$ & \\
\hline - PEG ,4L & $128(38.9)$ & $143(44.4)$ & \\
\hline \multicolumn{3}{|l|}{ Dosing, n (\%) } & 0.72 \\
\hline - Split dose 2 days & $208(63.2)$ & $207(64.3)$ & \\
\hline - Split dose same day & $33(10.0)$ & $28(8.7)$ & \\
\hline - Day before & $85(25.8)$ & $86(26.7)$ & \\
\hline Interval $\leq 5$ hours, $\mathrm{n}(\%)$ & $174(52.9)$ & $164(50.9)$ & 0.53 \\
\hline \multicolumn{3}{|l|}{ Instructions, n (\%) } & 0.56 \\
\hline
\end{tabular}

- Table 1 (Continuation)

\begin{tabular}{|c|c|c|c|}
\hline Variable & $\begin{array}{l}\text { Control } \\
(n=329)\end{array}$ & $\begin{array}{l}\text { Telephone } \\
(\mathrm{n}=322)\end{array}$ & $\begin{array}{l}P \\
\text { value }\end{array}$ \\
\hline - Oral & $30(9.1)$ & $31(9.6)$ & \\
\hline - Written & $184(55.9)$ & $183(56.8)$ & \\
\hline - Oral and written & $112(34.3)$ & $108(33.5)$ & \\
\hline Morning schedule, n (\%) & $235(71.4)$ & $216(67.1)$ & 0.30 \\
\hline \multicolumn{3}{|c|}{ Medical education provider, n (\%) } & 0.54 \\
\hline - Nurse & $93(28.3)$ & $87(27.0)$ & \\
\hline - Physician & $101(30.7)$ & $104(32.3)$ & \\
\hline - Secretary & $133(40.4)$ & $131(40.7)$ & \\
\hline $\begin{array}{l}\text { Complete colonoscopy rate, } \\
\mathrm{n}(\%)\end{array}$ & $170(51.7)$ & $165(51.2)$ & 0.91 \\
\hline
\end{tabular}

IQR, interquartile range; BMI, body mass index; ASA, American Society of Anesthesiologists; MCSP, magnesium citrate plus sodium picosulfate; PEG, polyethylene glycol.

Medical conditions associated with poor bowel cleansing but affecting $<5 \%$ of patients (cirrhosis, stroke, severe renal impairment, Parkinson disease or dementia) are not represented.

rated lesions in the telephone group ( $\triangleright$ Table 3 ). There was also a trend toward a higher distal adenoma detection rate and mean adenoma per patient in the telephone group ( $\triangleright$ Table 3 ).

For patients randomized to the intervention group, we compared patients who were successfully contacted with those who were impossible to reach (Table 1s). There were no significant differences in their baseline characteristics.

Analysis of bowel preparation success according to several risk factors revealed that the telephone intervention was particularly effective when the indication for the colonoscopy was symptoms. In contrast, we could not show any improvement in patients with diabetes mellitus, constipation or without abdominal or pelvic surgery ( $\triangleright$ Table 4 ).

Regarding the cost-effectiveness analysis, the mean duration of the telephone intervention was 8.1 minutes. The mean cost of the nurse-led telephone intervention was $€ 5.1$, including $15 \%$ of nonpersonnel costs. Considering an NNT of 18.9 telephone calls to prevent one bowel preparation failure, €96 would be spent in preventing one failure. Therefore, the implementation of the nurse-led telephone education would be costeffective, saving $€ 224$ per bowel preparation failure.

\section{Discussion}

This is the first multicenter trial to assess an educational intervention for improving bowel preparation in patients at high risk of poor bowel cleansing. In the whole group (ITT analysis), a telephone interview conducted by a trained nurse 24-48 hours before the colonoscopy did not show any significant benefit in bowel preparation quality in patients with previous bowel preparation failure. However, in patients who could actually be contacted by telephone and who received the educational inter- 
- Table 2 Outcome measures in the intention-to-treat and per-protocol analyses.

\begin{tabular}{|c|c|c|c|c|}
\hline Variable & Control $(n=329)$ & Telephone $(n=322)$ & ARD (95\%Cl) & $P$ value \\
\hline \multicolumn{5}{|c|}{ Successful bowel preparation, $\mathrm{n} / \mathrm{N}(\%)$} \\
\hline . ITT & $237 / 329(72.0)$ & $249 / 322(77.3)$ & $5.3(-1.4$ to 12.0$)$ & 0.12 \\
\hline - PP & $237 / 329(72.0)$ & $223 / 267(83.5)$ & 11.5 (4.9 to 18.1$)$ & 0.001 \\
\hline \multicolumn{5}{|c|}{ Colonoscopy attendance, $\mathrm{n} / \mathrm{N}(\%)$} \\
\hline - ITT & $302 / 329(91.8)$ & $303 / 322(94.1)$ & $2.3(-1.6$ to 6.2$)$ & 0.25 \\
\hline " PP & $302 / 329(91.8)$ & $263 / 267(98.5)$ & $6.7(3.4$ to 10.0$)$ & 0.001 \\
\hline \multicolumn{5}{|c|}{ BBPS score $\geq 2$ in all segments, $n / N(\%)$} \\
\hline - ITT & $237 / 302(78.5)$ & $249 / 303(82.2)$ & $3.7(-2.6$ to 10.0$)$ & 0.25 \\
\hline - PP & $237 / 302(78.5)$ & $223 / 263(84.8)$ & $6.3(0.0$ to 12.7$)$ & 0.05 \\
\hline \multicolumn{5}{|c|}{ Right colon BBPS score $\geq 2, n / N(\%)$} \\
\hline - ITT & $235 / 284(82.7)$ & $242 / 284(85.2)$ & $2.5(-3.6$ to 8.5$)$ & 0.42 \\
\hline - PP & $235 / 284(82.7)$ & $218 / 248(87.9)$ & $5.2(-0.8$ to 11.1$)$ & 0.10 \\
\hline \multicolumn{5}{|c|}{ Transverse colon BBPS score $\geq 2, \mathrm{n} / \mathrm{N}(\%)$} \\
\hline - ITT & $255 / 290(87.9)$ & 269/291 (92.4) & $4.5(-0.3$ to 9.3$)$ & 0.07 \\
\hline - PP & $255 / 290(87.9)$ & $235 / 253(92.9)$ & 5 (0.0 to 9.9$)$ & 0.05 \\
\hline \multicolumn{5}{|c|}{ Left colon BBPS score $\geq 2, n / N(\%)$} \\
\hline - ITT & $259 / 301(86.0)$ & $274 / 303(90.4)$ & 4.4 (-0.7 to 9.5$)$ & 0.10 \\
\hline - PP & $259 / 301(86.0)$ & $242 / 263(92.0)$ & $6(0.9$ to 11.1$)$ & 0.03 \\
\hline \multicolumn{5}{|c|}{ Complete colonoscopy, n/N (\%) } \\
\hline . ITT & $265 / 302(87.7)$ & $280 / 303(92.4)$ & $4.7(-0.1$ to 9.4$)$ & 0.06 \\
\hline - PP & $265 / 302(87.7)$ & $244 / 263(92.8)$ & 5 (0.2 to 9.9$)$ & 0.046 \\
\hline \multicolumn{5}{|c|}{ Adherence to low-fiber diet, $\mathrm{n} / \mathrm{N}$ (\%) } \\
\hline . ITT & $264 / 300(88.0)$ & $256 / 302(84.8)$ & $-3.2(-8.7$ to 2.2$)$ & 0.25 \\
\hline - PP & $264 / 300(88.0)$ & $222 / 262(84.7)$ & $-3.3(-9.0$ to 2.4$)$ & 0.26 \\
\hline \multicolumn{5}{|c|}{ Laxative intake > 75\%, n/N (\%) } \\
\hline . ITT & $294 / 301(97.7)$ & $296 / 300(98.7)$ & $1(-1.1$ to 3.1$)$ & 0.36 \\
\hline . PP & $294 / 301(97.7)$ & $256 / 260(98.5)$ & $0.8(-1.5$ to 3.1$)$ & 0.50 \\
\hline \multicolumn{5}{|c|}{ Interval ${ }^{*}<5$ hours, $\mathrm{n} / \mathrm{N}(\%)$} \\
\hline - ITT & $232 / 277(83.8)$ & $238 / 276(86.2)$ & $2.5(-3.5$ to 8.4$)$ & 0.42 \\
\hline - PP & $232 / 277(83.8)$ & $206 / 237$ (86.9) & $3.2(-2.9$ to 9.3$)$ & 0.31 \\
\hline
\end{tabular}

vention (per-protocol analysis), bowel preparation success increased by $11.5 \%$.

Two previous single-center RCTs investigated educational reinforcement by telephone. Liu et al. [11] demonstrated that telephone re-education on the day before the colonoscopy increased the rate of adequate bowel preparation (70.3\% vs. $81.6 \% ; P=0.001)$. Another RCT showed that educational intervention by telephone or SMS prior to colonoscopy improved bowel preparation in both groups compared with controls [12], without differences between the two interventions. Unlike these two RCTs, it is important to note that the present study included only patients who had previous unsuccessful bowel preparation, which has been identified as the most significant factor predicting poor bowel preparation, making our patients a truly difficult-to-prepare population. This could also explain the low telephone contact rate. In addition, our patients had higher rates of nonmodifiable risk factors for poor bowel preparation than the other studies, which included a nonselected 
- Table 3 Detected lesions

\begin{tabular}{|c|c|c|c|}
\hline Variable & Control $(n=329)$ & Telephone $(n=322)$ & $P$ value \\
\hline \multicolumn{4}{|c|}{ Mean adenoma per patient $(95 \% \mathrm{Cl})$} \\
\hline - ITT & 0.84 (0.66 to 1.03$)$ & 1.14 (0.89 to 1.4$)$ & 0.07 \\
\hline - PP & 0.84 (0.66 to 1.03$)$ & $1.1(0.84$ to 1.35$)$ & 0.11 \\
\hline \multicolumn{4}{|c|}{ Overall adenoma detection rate, $\mathrm{n} / \mathrm{N}(\%)$} \\
\hline - ITT & $117 / 302(38.7)$ & $130 / 303(42.9)$ & 0.30 \\
\hline - PP & $117 / 302(38.7)$ & $116 / 263(44.1)$ & 0.20 \\
\hline \multicolumn{4}{|c|}{ Proximal adenoma detection rate, $\mathrm{n} / \mathrm{N}(\%)$} \\
\hline - ITT & $78 / 302(25.8)$ & $94 / 303(31.0)$ & 0.16 \\
\hline - PP & $78 / 302(25.8)$ & $85 / 263(32.3)$ & 0.09 \\
\hline \multicolumn{4}{|c|}{ Distal adenoma detection rate, $\mathrm{n} / \mathrm{N}(\%)$} \\
\hline - ITT & $62 / 302(20.5)$ & $82 / 303(27.1)$ & 0.06 \\
\hline - PP & $62 / 302(20.5)$ & $71 / 263(27.0)$ & 0.07 \\
\hline \multicolumn{4}{|c|}{ Multiple adenoma ( $\geq 3), \mathrm{n} / \mathrm{N}(\%)$} \\
\hline . ITT & $29 / 302(9.6)$ & 45/303 (14.9) & 0.049 \\
\hline - PP & $29 / 302(9.6)$ & $39 / 263(14.8)$ & 0.06 \\
\hline \multicolumn{4}{|c|}{ Overall serrated detection rate, $n / N(\%)$} \\
\hline - ITT & $27 / 302(8.9)$ & $45 / 303(14.9)$ & 0.03 \\
\hline - PP & $27 / 302(8.9)$ & $40 / 263(15.2)$ & 0.02 \\
\hline \multicolumn{4}{|c|}{ Invasive neoplasia, n/N (\%) } \\
\hline - ITT & $5 / 302(1.7)$ & $6 / 303(2.0)$ & 0.77 \\
\hline - PP & $5 / 302(1.7)$ & $5 / 263(1.9)$ & 0.83 \\
\hline
\end{tabular}

population. Therefore, our intervention may be considered as a salvage strategy for difficult-to-prepare patients, and the apparent negative results of the intervention could be undermined by the characteristics of the included sample [6].

The abovementioned studies have several flaws. First, a strikingly low rate of adequate bowel cleansing in both the control and intervention groups was found [11] compared with large prospective studies in unselected populations [24]. Second, regular instructions provided by a nurse and colonoscopy in such a short time frame raises concerns about the quality of the standard education [11]. Third, these studies were focused on patients at low risk of poor bowel preparation, such as a screening population, which probably benefits less from this type of intervention. Fourth, the studies lacked important information such as the nonattendance rate or the contact rate for the telephone intervention. Finally, the results may not be extrapolated to Western populations, and the single-center settings prevent external validation.

Inadequate bowel preparation is a major burden requiring innovative solutions. In our trial, we decided to include a number of measures that have proven efficacy in bowel cleansing, such as split dosing, a short interval between the final laxative dose and the colonoscopy, and specific verbal and written instructions explaining the bowel preparation. We chose $4 \mathrm{~L}$ of PEG rather than low-volume PEG, following the evidence from a recent RCT in patients with bowel preparation failure [19]. Furthermore, pump irrigation, which has been proven effective for increasing bowel cleansing [25], was included for all patients in the trial. Although other approaches may be considered, such as additional laxative intake the same day or the next day following the examination, or the administration of enemas through the colonoscope, the implementation of these strategies in clinical practice is limited by scheduling problems.

Despite the heterogeneity of the interventions for reinforcing medical education before colonoscopy, in general they have been shown to improve cleansing quality [26]. In our study, we applied our best knowledge to deliver instructions at the colonoscopy request time, such as a clearly written leaflet and a nurse-led face-to-face interview to individualize bowel preparation. In addition, the telephone interview shortly before the colonoscopy appointment was conducted by an experienced nurse because it allows direct bidirectional communication that may increase the comprehension and adherence to instructions. The timing of the telephone call shortly before the colonoscopy appointment was chosen because laxative intake and dosing are the most important factors affecting the quality of bowel preparation [27]. Telephone intervention planned well in advance of the colonoscopy may have increased the adherence to the recommended diet. However, recent studies have shown that diet restrictions are not so important in increasing bowel preparation effectiveness $[18,28]$, and the reminder about laxative intake may fade over time.

A face-to-face visit to educate patients may be at least as effective, if not better than a telephone call. However, visits require more resources, are more rigid in terms of patient and nurse availability, and have more scheduling limitations. These issues would probably make a face-to-face intervention more expensive and more difficult to implement than a telephone call. In our trial, an initial face-to-face visit was required in order to conduct the RCT. During this visit, the investigators educated patients regarding bowel preparation instructions. Such education, given by experienced investigators, may have reduced the potential benefit of the telephone intervention.

As expected, we found that the benefit of this telephone intervention was limited to those patients who could be contacted, with a positive effect on cleansing quality overall and per segment; $17 \%$ of the patients could not be contacted and thus did not benefit. We did not find significant differences in the baseline characteristics between patients who were contacted and those who were not. However, information about the socioeconomic status that may have influenced the applicability of the telephone intervention was not analyzed.

The telephone intervention was particularly effective in patients who were referred for symptoms, whereas there was no significant benefit in screening or surveillance patients. It is well known that the baseline bowel preparation success rate is higher in patients referred for screening colonoscopy, as these patients usually have fewer comorbid conditions. The past experience of 
- Table4 Bowel preparation success by subgroups of risk factors.

\begin{tabular}{|c|c|c|c|c|c|}
\hline Variable & Control, n/N (\%) & Telephone, n/N (\%) & ARD $(95 \% \mathrm{Cl})$ & $\mathbf{L R}^{*}$ & Corrected $P$ value \\
\hline \multicolumn{4}{|l|}{ Diabetes mellitus } & 4.85 & 0.35 \\
\hline - Yes & $52 / 79(65.8)$ & $42 / 61(68.9)$ & $3(-12.6$ to 18.0$)$ & & \\
\hline - No & $185 / 250(74.0)$ & $207 / 261(79.3)$ & $5.3(-2.0$ to 12.6$)$ & & \\
\hline \multicolumn{4}{|c|}{ Abdominal/pelvic surgery } & 5.08 & 0.32 \\
\hline - Yes & $99 / 138(71.7)$ & $96 / 135(71.1)$ & $-0.6(-11.3$ to 10.0$)$ & & \\
\hline - No & $138 / 191(72.3)$ & $153 / 187(81.8)$ & $9.6(1.1$ to 17.9$)$ & & \\
\hline \multicolumn{4}{|l|}{ Constipation } & 6.8 & 0.13 \\
\hline - Yes & $83 / 123(67.5)$ & $89 / 125(71.2)$ & $3.7(-7.7$ to 15.0$)$ & & \\
\hline - No & $153 / 202(75.7)$ & $159 / 196(81.1)$ & $5.4(-2.7$ to 13.4$)$ & & \\
\hline \multicolumn{4}{|l|}{ Indication } & 17.22 & 0.007 \\
\hline - Screening & $71 / 87(81.6)$ & $71 / 85(83.5)$ & $1.9(-9.6$ to 13.3$)$ & & \\
\hline - Surveillance & $76 / 96(79.2)$ & $71 / 95(74.7)$ & $-4.4(-16.3$ to 7.5$)$ & & \\
\hline - Symptoms & $89 / 145(61.4)$ & $107 / 142(75.4)$ & 14 (3.2 to 24.3$)$ & & \\
\hline
\end{tabular}

patients undergoing surveillance colonoscopy could also affect their baseline adherence to bowel preparation instructions, reducing the benefit of the telephone intervention [24].

In the present study, more patients with multiple adenomas $(\geq 3)$ and more serrated lesions were detected in the intervention group. These findings may be explained by the better bowel cleansing found in the per-protocol analysis in the transverse and left colon segments and more complete colonoscopies in the telephone group. It is also worth mentioning that the lesions detected in the colonoscopy, including 11 cancers (1.7\%), had been missed during the index colonoscopy. It is important to note that poor bowel cleansing prevents both complete colonoscopy and mucosal visualization in the explored colon. These results illustrate the importance of repeating colonoscopies in cases of inadequate cleansing [29].

Another aspect is that the cost-effectiveness analysis revealed that the nurse-led telephone education was cost-saving.

Our study has several strengths. First, it is the first RCT to evaluate an educational intervention in patients with a high risk of poor bowel preparation. Second, the multicenter design favors the generalization of the results. Third, we used the BBPS, which is the most thoroughly validated scale for evaluating the quality of bowel preparation, allowing comparisons between studies. Fourth, we provided a telephone noncontact rate, which measured the applicability of the intervention. Finally, to our knowledge, the best bowel cleansing recommendations (not including the telephone intervention material) were provided to all included patients in the trial. This multifactorial approach has previously been shown to be beneficial [30]. In that sense, we may consider that in patients with a past history of poor bowel cleansing, it would be difficult to exceed the rate of $85 \%$ successful cleansing.
Our study has some limitations. First, the educational intervention only had a positive effect when patients were successfully contacted by telephone. However, this fact is also proof of the benefit of such an intervention. Second, to standardize the intervention, we centralized all telephone calls at the coordinating center in Barcelona. However, we believe that from a pragmatic point of view, this intervention should be performed by local centers. In any case, it is interesting to note that this contact rate $(83 \%)$ was similar to another single-center study from our group [31]. Third, some secondary outcomes were planned a priori but were not listed in the registered protocol at ClinicalTrials.gov. Fourth, bowel stenosis may have been considered as an exclusion criterion as it may impair bowel cleansing; however, only two colonoscopies were incomplete due to malignant stenosis. Fifth, patients with colonoscopy appointment on Mondays were contacted on Fridays ( 72 hours before the colonoscopy). This situation may have influenced the contact rate. Sixth, the positive impact of our educational intervention was mainly because of the increase in the attendance rate and not because of significantly better bowel cleansing. Although reminder tools could deliver similar benefits in the general population and be cost-effective [12], it is unclear whether the same effect is achieved in difficult-to-prepare patients. It would be interesting to investigate whether the combination of a telephone call and an SMS as a backup contact method would increase the attendance rate. Finally, we might also face a "ceiling effect" where reinforced education may not improve the bowel preparation efficacy over a threshold, particularly in patients with impaired bowel peristalsis.

In conclusion, this multicenter trial showed that a nurse-led telephone educational intervention within 48 hours before colonoscopy did not reach a significant increase in bowel preparation 
success in patients with previous failure. However, in the $83 \%$ of patients who were contactable and received the intervention, the bowel preparation success rate was substantially improved.

\section{Acknowledgments}

This paper was presented as an oral presentation at the endoscopy annual meeting of the Sociedad Española de Endoscopia Digestiva 2019, Alicante, Spain. It was also presented as an oral presentation at the gastrointestinal annual meeting of the Asociación Española de Gastroenterología 2019, Madrid, Spain. Finally, it was presented as a poster at United European Gastroenterology Week (UEGW) 2019, Barcelona, Spain.

This study was supported by grants from the Asociación Española de Gastroenterología and the Societat Catalana de Digestología. The authors gratefully acknowledge the valuable contributions from all registered nurses who participated in the study: Roberto Borja Manzanares, Cristina Lao, Mariluz Mestre, and Montserrat Cornet. In addition, we express our gratitude to Springer Nature for editorial assistance. We also show our appreciation to both Lluis Mateu Castell and Marc Alegret Antonijuan for their collaboration in the study by collecting data. Finally, we thank Francesc Cots Reguant for his help with the cost-effectiveness analysis.

\section{Competing interests}

Marco Antonio Álvarez-Gonzáles has received honoria from Norgine Ldt. for advisory board attendance, speaking and teaching and from Casen-Recordati for speaking and teaching.

Clinical trial

Trial Registration: ClinicalTrials.gov | Registration number (trial ID): NCT03055689 | Type of study: prospective, randomized, multi-center study

References

[1] Kaminski MF, Thomas-Gibson S, Bugajski M et al. Performance measures for lower gastrointestinal endoscopy: a European Society of Gastrointestinal Endoscopy (ESGE) quality improvement initiative. United Eur Gastroenterol J 2017; 5: 309-334

[2] Mahmood S, Farooqui SM, Madhoun MF. Predictors of inadequate bowel preparation for colonoscopy: a systematic review and meta-analysis. Eur J Gastroenterol Hepatol 2018; 30: 819-826

[3] Clark BT, Rustagi T, Laine L. What level of bowel prep quality requires early repeat colonoscopy: systematic review and meta-analysis of the impact of preparation quality on adenoma detection rate. Am J Gastroenterol 2014; 109: 1714-1723

[4] Rex DK, Imperiale TF, Latinovich DR et al. Impact of bowel preparation on efficiency and cost of colonoscopy. Am J Gastroenterol 2002; 97: 1696-1700

[5] Saltzman JR, Cash BD, Pasha SF et al. Bowel preparation before colonoscopy. Gastrointest Endosc 2015; 81: 781-794

[6] Ben-Horin S, Bar-Meir S, Avidan B. The outcome of a second preparation for colonoscopy after preparation failure in the first procedure. Gastrointest Endosc 2009; 69: 626-630
[7] Ness RM, Manam R, Hoen $\mathrm{H}$ et al. Predictors of inadequate bowel preparation for colonoscopy. Am J Gastroenterol 2001; 96: 17971802

[8] Hassan C, East J, Radaelli F et al. Bowel preparation for colonoscopy: European Society of Gastrointestinal Endoscopy (ESGE) Guideline update 2019. Endoscopy 2019; 45: 142-155

[9] Lebwohl B, Wang TC, Neugut Al. Socioeconomic and other predictors of colonoscopy preparation quality. Dig Dis Sci 2010; 55: 2014-2020

[10] Jones RM, Woolf SH, Cunningham TD et al. The relative importance of patient-reported barriers to colorectal cancer screening. Am J Prev Med 2010; 38: 499-507

[11] Liu X, Luo H, Zhang L et al. Telephone-based re-education on the day before colonoscopy improves the quality of bowel preparation and the polyp detection rate: a prospective, colonoscopist-blinded, randomised, controlled study. Gut 2014; 63: 125-130

[12] Lee Y], Kim ES, Choi JH et al. Impact of reinforced education by telephone and short message service on the quality of bowel preparation: a randomized controlled study. Endoscopy 2015; 47: 1018-1027

[13] Guo X, Yang Z, Zhao L et al. Enhanced instructions improve the quality of bowel preparation for colonoscopy: a meta-analysis of randomized controlled trials. Gastrointest Endosc 2017; 85: 90-97.e6

[14] Johnson DA, Barkun AN, Cohen LB et al. Optimizing adequacy of bowel cleansing for colonoscopy: recommendations from the US Multi-Society Task Force on Colorectal Cancer. Am J Gastroenterol 2014; 109: 1528-1545

[15] Clark BT, Protiva P, Nagar A et al. Quantification of adequate bowel preparation for screening or surveillance colonoscopy in men. Gastroenterology 2016; 150: 396-405

[16] Dumonceau J-M, Riphaus A, Schreiber F et al. Non-anesthesiologist administration of propofol for gastrointestinal endoscopy: European Society of Gastrointestinal Endoscopy, European Society of Gastroenterology and Endoscopy Nurses and Associates Guideline - updated June 2015. Endoscopy 2015; 47: 1175-1189

[17] Hassan C, Bretthauer M, Kaminski MF et al. Bowel preparation for colonoscopy: European Society of Gastrointestinal Endoscopy (ESGE) guideline. Endoscopy 2013; 45: 142-150

[18] Alvarez-Gonzalez MA, Pantaleon MA, Flores-Le RouxJA et al. Randomized clinical trial: a normocaloric low-fiber diet the day before colonoscopy is the most effective approach to bowel preparation in colorectal cancer screening colonoscopy. Dis Colon Rectum 2019; 62: 491-497

[19] Gimeno-García AZ, Hernandez G, Aldea A et al. Comparison of two intensive bowel cleansing regimens in patients with previous poor bowel preparation: a randomized controlled study. Am J Gastroenterol 2017; 112: 951-958

[20] Calderwood AH, Jacobson BC. Comprehensive validation of the Boston Bowel Preparation Scale. Gastrointest Endosc 2010; 72: 686-692

[21] Gimeno-García AZ, Baute JL, Hernandez G et al. Risk factors for inadequate bowel preparation: a validated predictive score. Endoscopy 2017; 49: 536-543

[22] Dik VK, Moons LMG, Hüyük M et al. Predicting inadequate bowel preparation for colonoscopy in participants receiving split-dose bowel preparation: development and validation of a prediction score. Gastrointest Endosc 2015; 81: 665-672

[23] Diari Oficial de la Generalitat de Catalunya ORDRE SLT/165/2018. contraprestació de l'atenció hospitalària i especialitzada; 2018: Available from: https://portaldogc.gencat.cat/utilsEADOP/PDF/ 7723/1697258.pdf 1-10

[24] Mangas-Sanjuan C, Santana E, Cubiella J et al. Variation in colonoscopy performance measures according to procedure indication. Clin Gastroenterol Hepatol 2020; 18: 1216-1223.e2

[25] Rigaux J, Juriens I, Devière J. A novel system for the improvement of colonic cleansing during colonoscopy. Endoscopy 2012; 44: 703-706 
[26] Hernández G, Gimeno-García AZ, Quintero E. Estrategias para optimizar la calidad de la limpieza colónica. Gastroenterol Hepatol 2019; 42: 326-338

[27] Radaelli F, Paggi S, Hassan C et al. Split-dose preparation for colonoscopy increases adenoma detection rate: a randomised controlled trial in an organised screening programme. Gut 2017; 66: 270-277

[28] Gimeno-García AZ, de la Barreda Heuser R, Reygosa C et al. Impact of a 1-day versus 3-day low-residue diet on bowel cleansing quality before colonoscopy: a randomized controlled trial. Endoscopy 2019; 51: $628-636$
[29] Chokshi RV, Hovis CE, Hollander T et al. Prevalence of missed adenomas in patients with inadequate bowel preparation on screening colonoscopy. Gastrointest Endosc 2012; 75: 1197-1203

[30] Alvarez-Gonzalez MA, Flores-Le Roux AA, Seoane A et al. Efficacy of a multifactorial strategy for bowel preparation in diabetic patients undergoing colonoscopy: a randomized trial. Endoscopy 2016; 48: 1003-1009

[31] Seoane Urgorri A, Font Lagarriga X, Pérez Berbegal R et al. Educational telephone intervention by endoscopy nurse. Impact on the adherence of outpatient colonoscopy. Endoscopy 2019; 51: (Suppl. 04): S125 